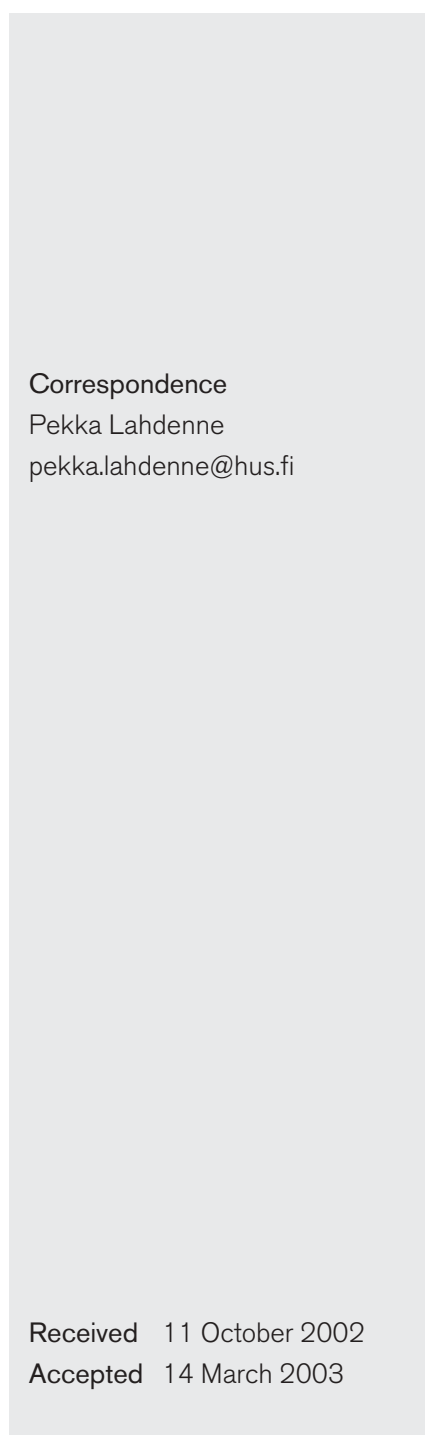

\title{
Improved serodiagnosis of erythema migrans using novel recombinant borrelial BBK32 antigens
}

\author{
Pekka Lahdenne, ${ }^{1}$ Jaana Panelius, ${ }^{2}$ Harri Saxen, ${ }^{1}$ Tero Heikkilä, ${ }^{1}$ \\ Heidi Sillanpää, ${ }^{2}$ Miikka Peltomaa, ${ }^{3,4}$ Maja Arnez, ${ }^{5}$ Hans-Iko Huppertz ${ }^{6}$ \\ and Ilkka J. T. Seppälä2,7 \\ ${ }^{1}$ Hospital for Children and Adolescents, University of Helsinki, Stenbäckinkatu 11, 00290 Helsinki, \\ Finland \\ ${ }^{2}$ Department of Bacteriology and Immunology, Haartman Institute, Haartmaninkatu 3, 00014 \\ Helsinki, Finland \\ 3,7 Department of Otolaryngology ${ }^{3}$ and Laboratory Diagnostics ${ }^{7}$, Helsinki University Central Hospital, \\ 00290 Helsinki, Finland \\ ${ }^{4}$ Division of Rheumatology, Allergy and Immunology, Massachusetts General Hospital, Harvard \\ Medical School, Charlestown, MA 02129, USA \\ ${ }^{5}$ Department of Infectious Diseases, University Medical Center Ljubljana, Slovenia \\ ${ }^{6}$ Professor-Hess-Children's Hospital Bremen and Children's Hospital of the University of Würzburg, \\ Germany
}

\begin{abstract}
The performances of recombinant borrelial BBK32 proteins as antigens in the serology of erythema migrans (EM) were evaluated in an ELISA. Serum samples were obtained from 75 patients from different geographic areas where three borrelial species, Borrelia burgdorferi sensu stricto, Borrelia afzelii or Borrelia garinii, cause Lyme borreliosis. Antibodies to variant BBK32 proteins were compared with anti-flagella or with anti-IR 6 peptide antibodies. In IgG ELISA at presentation of EM, $65 / 75$ (87\%) patients had antibodies to one or more variants of BBK32, 29/75 (39\%) had antibodies to flagella and 29/75 (39\%) had antibodies to the VlsE IR6 peptide antigen. The immunoreactivity against variant BBK32 proteins differed in patients from different geographic regions. The present results suggest that the BBK32 proteins used in combination or in parallel may improve the laboratory diagnosis of EM.
\end{abstract}

\section{INTRODUCTION}

Lyme borreliosis (LB) is caused by spirochaetes belonging to the complex Borrelia burgdorferi sensu lato, and is characterized by multistage skin, joint, neurological and cardiac manifestations (Steere, 2001). The earliest and most common manifestation of LB is erythema migrans (EM), which appears at the site of the tick bite days to weeks after exposure. Tick bites may easily be unrecognized, and the clinician has to rely on the appearance of the skin lesion. In a routine clinical setting, EM is considered to be pathognomonic for early LB. The classical appearance of EM is an enlarging, ringlike erythema with a central clearing. However, early in the course of LB, atypical lesions may occur and cause diagnostic problems (Oksi et al., 2001; Smith et al., 2002). The gold standard for the diagnosis of early LB is microbiological confirmation of LB by culture from biopsies taken from EM

Abbreviations: EM, erythema migrans; LB, Lyme borreliosis. lesions and/or from blood. PCR-based methods can also be used to detect B. burgdorferi DNA in skin biopsies. However, in routine clinical practice, these methods are not feasible. Therefore, accurate detection of borrelial infection requires other laboratory tests with sufficient sensitivities and specificities.

The drawback of the current serologic tests during early LB is their low sensitivity. The sensitivity of the IgM or IgG ELISA using borrelial flagella or whole-cell lysate antigens seldom exceeds 40-50 \% (Mitchell et al., 1994; Aguero-Rosenfeld et al., 1996; Nowakowski et al., 2001; Vaz et al., 2001). New recombinant borrelial proteins or synthetic peptides have been tested in LB serology with promising results (Liang et al., 1999; Gomes-Solecki et al., 2001). We recently evaluated recombinant BBK32 (rBBK32) protein antigens in serologic assays for LB (Heikkilä et al., 2002). The rBBK32 protein appeared to be a useful antigen in early and late LB, especially in IgG serology, but not in IgM serology. However, our recent 
study suggested species-specific variations in the immune responses to the variant BBK32 proteins in early LB (Heikkilä et al., 2002). The rBBK32 antigen originating from a local $B$. afzelii strain appeared to be superior to the other heterologous rBBK32 variants.

In the present study, we have evaluated the performances of three variant BBK32 antigens in the IgG serology of EM in four different geographic areas where three pathogenic borrelial species, B. burgdorferi sensu stricto, B. afzelii and $B$. garinii, are the common aetiologic agents of LB. Antibodies to variant $\mathrm{rBBK} 32$ proteins were compared with antibodies to commercial flagella antigens (Dako) and to a recently described peptide antigen, VlsE $\mathrm{IR}_{6}$ (Liang et al., 1999).

\section{METHODS}

Patients. Serum samples were collected from patients with clinically documented or culture- or PCR-confirmed EM from Finland, Germany, Slovenia and the USA. All 23 Finnish sera were from culture $(n=18)$ and/or from PCR $(n=21)$ proven patients taken at the time of diagnosis (acute) and 1-3 months after treatment (convalescent) (Heikkilä et al., 2002). In the 23 patients with EM, genotyping by PCR analysis (Junttila et al., 1999) showed B. afzelii in 17 and B. garinii in 4 of the skin biopsies. In two biopsies, genotyping was not successful. As part of a regional study on LB, German samples were collected in Northern Bavaria (Huppertz et al., 1999). Sera were collected from 22 patients with physician-diagnosed EM at the time of diagnosis and during the convalescence phase (Huppertz et al., 1999). The clinical diagnosis of EM was confirmed by especially trained physicians. In addition, sera were collected from 10 US patients with culture-positive $\mathrm{EM}$ at the time of diagnosis and during convalescence 3-4 weeks after treatment (Vaz et al., 2001). Serum samples were also available from 20 Slovenian patients in the acute stage of EM. Two out of nine Slovenian patients from whom skin biopsies were taken were culture-positive $(B$. burgdorferi sensu lato) (Strle et al., 1996). The diameter of EM lesions in the Finnish, German or US patients varied between 4 and $27 \mathrm{~cm}$ (median $10 \mathrm{~cm}$ ), 5 and $23 \mathrm{~cm}$ (median $10 \mathrm{~cm}$ ) or 6 and $21 \mathrm{~cm}$ (median $10 \mathrm{~cm}$ ), respectively. In the 20 Slovenian patients, the size of the EM lesions was not available, but the duration of EM prior to the diagnosis was from 1 to 30 days (median 7 days). All patients with EM were treated with oral antimicrobials.

Serum samples from 40 Finnish healthy blood donors were used as negative controls and to define the cut-off value for all ELISAs (cutoff $=$ mean plus $3 \mathrm{SD}$ ).
ELISA. $b b k 32$ genes were cloned and sequenced from Finnish isolates of borrelial species representing B. afzelii, B. garinii and B. burgdorferi sensu stricto, as described previously (Heikkilä et al., 2002). The respective rBBK32 antigens were produced as glutathione-S-transferase-BBK32 fusion proteins, and used as separate antigens in ELISA. The IgG BBK32 ELISAs were performed as described earlier (Heikkilä et al., 2002). Briefly, serum samples were diluted $1: 100$ in $5 \mathrm{mg} \mathrm{BSA} \mathrm{ml}^{-1}$ in 0.155 M NaCl-0.04\% Tween 20 buffer (BSA-NaCl-Tween). Alkalinephosphatase-conjugated rabbit anti-human IgG (Jackson Immuno Research Laboratories) 1:5000 in BSA-NaCl-Tween was used as the secondary antibody. The reactions were visualized with 4-nitrophenylphosphate (Boehringer Mannheim), $1 \mathrm{mg} \mathrm{ml}^{-1}$ in diethanolamine buffer, $\mathrm{pH} 10 \cdot 0$. The $\mathrm{OD}_{405}$ measurements were made after 10 20 min using a Multiscan photometer (Thermo Labsystems). The specificity of the BBK32 ELISA has been previously analysed with serum samples from patients with syphilis $(n=10)$, systemic lupus erythematosus $(n=8)$, rheumatoid factor positivity $(n=8)$, antistreptolysin positivity $(n=8)$, Epstein-Barr virus infection $(n=10)$ and from healthy blood donors $(n=20)$. The total specificity of the BBK32 ELISAs with these samples was $93 \%$ (Heikkilä et al., 2002).

ELISAs for anti-flagella antibodies were performed as described earlier (Seppälä et al., 1994). Briefly, IgM or IgG antibodies against B. burgdorferi were measured with the commercial flagellin-based ELISA kit K0416 (Dako) modified by end point titration of the antibodies. An end point titre was obtained at an optical density level determined by the cut-off control provided with the kit. The cut-off control material conformed with the level of the mean +3 SD of the control samples from healthy blood donors living in southern Finland, an area endemic for LB.

The synthetic peptide antigen VlsE $\mathrm{IR}_{6}$ was produced in the Core Facility of Protein Chemistry at the Haartman Institute, University of Helsinki. The amino acid sequence of $\mathrm{IR}_{6}$ corresponded to the recently published invariable region 6 of the VlsE gene (Liang et al., 1999). For IgG ELISA with the $\mathrm{IR}_{6}$ peptide, the plate wells (Greiner) were initially coated with $0.05 \mu \mathrm{g}$ streptavidin (Boehringer Mannheim) in PBS overnight at $+4{ }^{\circ} \mathrm{C}$. After washing, $20 \mathrm{ng}$ per well of the aminoterminally biotinylated peptide in PBS-0.04\% Tween was added. The subsequent steps were performed as described above.

\section{RESULTS AND DISCUSSION}

\section{Antibodies at diagnosis}

At presentation of EM, 65 of the 75 (87\%) patients had IgG antibodies to one or more variants of rBBK32, 29/75 (39\%) had antibodies to flagella, and 29/75 (39\%) had antibodies to the $\mathrm{IR}_{6}$ peptide antigen (Table 1; Fig. 1). The majority of

Table 1. Number of positive sera (\%) for any of the three BBK32 variant antigens (BBK32-all), IR $\mathrm{R}_{6}$ peptide antigen and for flagella antigen (Dako) in patients with EM from Finland (FIN), Germany (GER), the USA and Slovenia (SLO)

\begin{tabular}{|c|c|c|c|c|c|c|c|c|c|}
\hline \multirow[t]{2}{*}{ Antigen } & \multicolumn{5}{|c|}{ At diagnosis } & \multicolumn{4}{|c|}{ At convalescence } \\
\hline & $\begin{array}{c}\text { FIN } \\
n=23\end{array}$ & $\begin{array}{c}\text { GER } \\
n=22\end{array}$ & $\begin{array}{c}\text { USA } \\
n=10\end{array}$ & $\begin{array}{c}\text { SLO } \\
n=20\end{array}$ & $\begin{array}{l}\text { Total } \\
n=75\end{array}$ & $\begin{array}{c}\text { FIN } \\
n=23\end{array}$ & $\begin{array}{c}\text { GER } \\
n=22\end{array}$ & $\begin{array}{c}\text { USA } \\
n=10\end{array}$ & $\begin{array}{c}\text { Total } \\
n=55\end{array}$ \\
\hline BBK32-all-IgG & $17(74)$ & $21(95)$ & $10(100)$ & $17(82)$ & $65(87)$ & $15(65)$ & $15(68)$ & $10(100)$ & $40(73)$ \\
\hline IR6-IgG & $7(30)$ & $7(32)$ & $4(40)$ & $11(55)$ & $29(39)$ & $6(26)$ & $6(27)$ & $7(70)$ & $19(35)$ \\
\hline Flagella-IgG & $6(26)$ & $9(41)$ & $4(40)$ & $10(50)$ & $29(39)$ & $6(26)$ & $11(50)$ & $8(80)$ & $25(45)$ \\
\hline Flagella-IgM & $3(13)$ & $8(36)$ & $2(20)$ & $9(45)$ & $22(29)$ & $4(17)$ & $7(32)$ & $8(80)$ & $19(34)$ \\
\hline Flagella-IgM or IgG & $6(26)$ & $13(59)$ & $4(40)$ & $12(60)$ & $35(46)$ & $8(35)$ & $15(68)$ & $10(100)$ & $33(60)$ \\
\hline
\end{tabular}



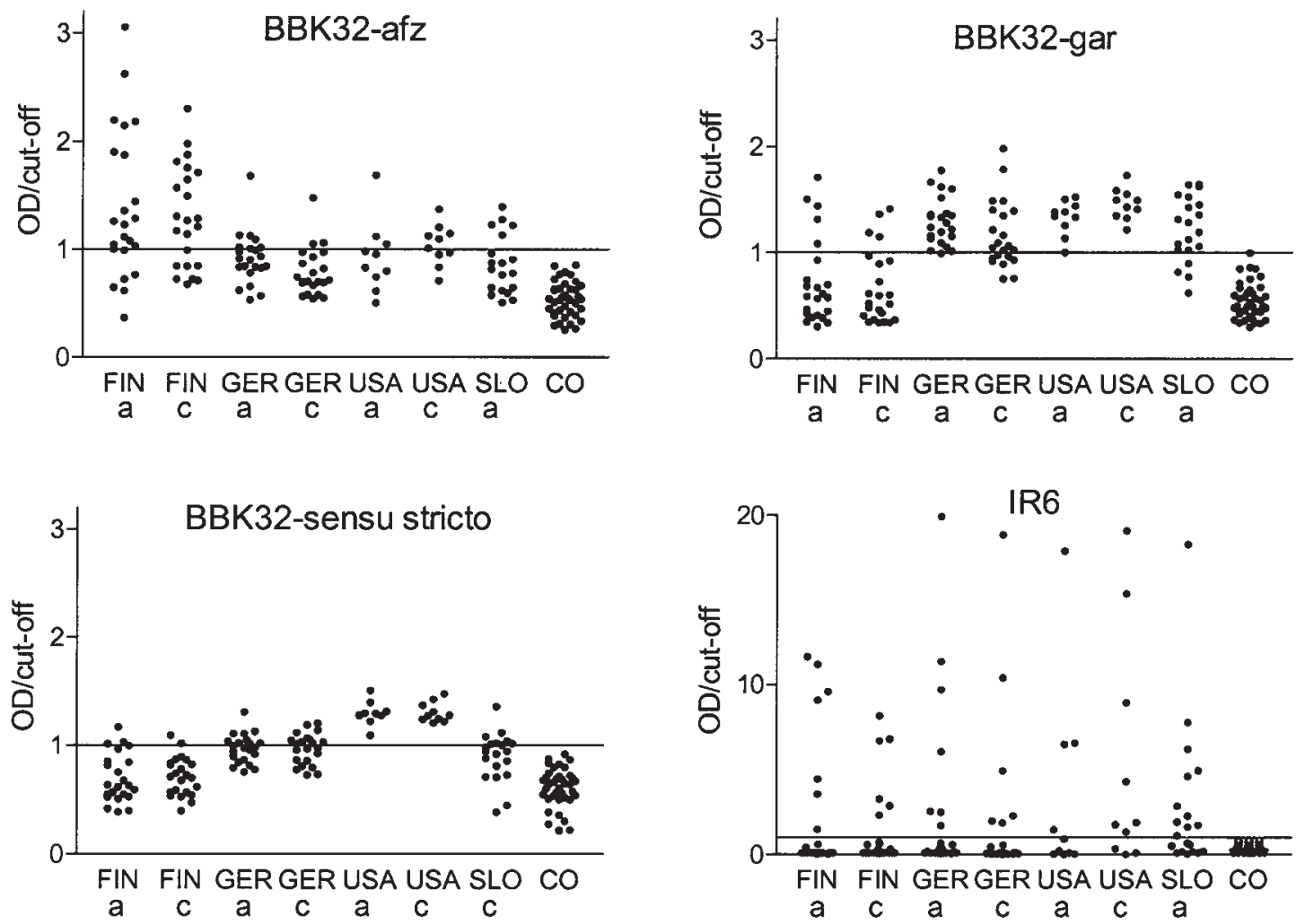

Fig. 1. ELISA OD/cut-off values of patients with EM from Finland (FIN), Germany (GER), the USA and Slovenia (SLO). Serum samples were taken at diagnosis (a) and after antibiotic treatment in convalescence (c). IgG antibodies to rBBK32 from B. afzelii (BBK32-afz), B. garinii (BBK32-gar), B. burgdorferi sensu stricto (BBK32-sensu stricto) and to IR6 peptide were assessed. Control samples (CO) were from 40 healthy blood donors. The level of positivity for OD/cut-off values $(>1)$ is indicated with a horizontal line.

patients from Finland had antibodies to rBBK32 from $B$. afzelii, whereas, in the German and Slovenian sera, the most sensitive antigen was rBBK32 from B. garinii. All 10 samples from the USA reacted positively with BBK32 from $B$. burgdorferi sensu stricto and from B. garinii. In total, 31 of the $75(41 \%)$ patients were positive for rBBK32 from $B$. afzelii, 52/75 (69\%) were positive for rBBK32 from B. garinii, and 30/75 (40\%) were positive for rBBK32 from B. burgdorferi sensu stricto. All the control samples were negative for rBBK32 variant antigens.

The proportion of patients with IgM anti-flagella antibodies at the time of diagnosis varied from 13 to $45 \%$ in the different regions. Of the total of 75 patients, $29 \%$ had IgM anti-flagella antibodies. IgM and/or IgG anti-flagella antibodies were detected in $46 \%$ of the patients (Table 1).

\section{Antibodies in the convalescent phase}

Forty of the 55 patients ( $73 \%$ ) in the convalescent phase had IgG antibodies to one or more rBBK32s, 25/55 (45\%) had antibodies to flagella and 19/55 (35\%) had antibodies to the $\mathrm{IR}_{6}$ peptide. The pattern of seropositivity to variant rBBK32s in the convalescent and the acute sera was similar. In the convalescence samples from Finland and Germany, the overall rate of IgG antibody positivity to rBBK32s had slightly decreased from that at diagnosis (Table 1; Fig. 1).

Recent reports from the USA and Europe (Oksi et al., 2001; Smith et al., 2002) have shown that the diagnosis of EM solely on clinical grounds may be difficult. During the first few days of infection, the pathognomonic expanding peripheral erythema with a central clearing appears to be far less common than lesions with homogeneous redness (Oksi et al., 2001; Smith et al., 2002). This implies that EM may be confused with other similar rashes, e.g. insect bites, etc. In order to avoid over- and underdiagnosis, there should be laboratory methods that would support or confirm the clinical diagnosis. We have previously shown that sera from the majority of Finnish patients with EM were positive for the rBBK32 antigen (Heikkilä et al., 2002). In the present study, we have expanded our studies to EM patients from different countries. The high rate of antibodies to one or more variants of rBBK32 suggests that these antigens might be used universally and already at presentation in the serology of EM.

The immunoreactivity against variant rBBK32 proteins diverged in the sera of EM patients from different countries. The species specificity of the antibodies corresponded well 
with the known aetiology of Finnish (B. afzelii) and American (B. burgdorferi sensu stricto) EM. We have recently reported that the sequence similarity of BBK32 between $B$. afzelii and B. garinii or B. burgdorferi sensu stricto is approximately $70 \%$, whereas the BBK32 proteins of B. garinii and B. burgdorferi sensu stricto are 93-100\% identical (Heikkilä et al., 2002). It is evident that the differences in the antibody levels of patients from different areas may be explained by sequence heterogeneity of the BBK32 proteins, especially that between $B$. afzelii and the other borrelial species. This implies that in regions where LB is caused by different species of $B$. burgdorferi sensu lato, variant BBK32 antigens are probably needed to cover all the EM cases.

At the time of diagnosis of EM, the duration of the disease correlates with the antibody positivity in the serum. Patients with a longer duration of EM appear to be more frequently positive for anti-B. burgdorferi antibodies (Aguero-Rosenfeld et al., 1996). This was also demonstrated in our study with the flagella antigen: convalescent sera contained antibodies more often than sera taken at presentation of EM. However, in clinical practice, diagnostic and therapeutic options of EM have to be considered at presentation. The present study suggests that the sensitivity of the rBBK32 ELISA in EM may be superior to that of the routinely used flagella or the new peptide antigen VlsE $\mathrm{IR}_{6}$.

The VlsE $I_{6}$ peptide has recently been shown to be a highly sensitive and specific antigen in the serology of LB (Liang et al., 1999). In disseminated LB, our results concur with the published data (unpublished). However, the present results indicate that in EM, IgG IR ${ }_{6}$ ELISA may be less sensitive. In our series, the immunoreactivity to $\mathrm{IR}_{6}$ did not differ significantly between patients with culture- or PCRconfirmed EM and clinically diagnosed EM. At presentation of EM, the overall seropositivity rate to $\mathrm{IR}_{6}$ was $39 \%$. This result differs from recent data by Liang et al. (1999), who reported $74-87 \%$ sensitivity in specimens from cultureconfirmed EM patients. A possible explanation for this discrepancy is that the serum samples in the present study were collected earlier during the course of LB than in the study by Liang et al. (1999). It is evident that further analyses of $\mathrm{IR}_{6}$ sequences and reactivity with human LB sera, in both IgM and IgG serology, in European conditions are also needed.

The BBK32 protein is a borrelial lipoprotein preferentially expressed in vivo. Expression of bbk32 is detectable in spirochaetes during tick feeding even before transmission to the host (Fikrig et al., 2000). Thus if the spirochaetes gain access to the skin of a human, there may be an early antibody response. Currently, it is not known whether bbk32 expression is down-regulated later during the course of infection. If EM is treated early on with antibiotics, antibodies to BBK32 might be useful markers of disease activity or response to therapy of EM. This hypothesis is supported by our observation that the proportion of anti-BBK32 antibodypositive samples was slightly lower at convalescence than at the acute phase. However, this decrease was only minor.
Therefore, further research with a greater number of samples is needed to confirm this possibility. So far, none of the current or novel Lyme disease tests seem to be able to differentiate active infection from a previous immune response.

In conclusion, this study with sera from epidemiologically diverse regions provides supporting evidence that the BBK32 proteins may be useful antigens in EM serology (Heikkilä et al., 2002). At presentation of EM, the sensitivity of the BBK32 ELISAs appeared better than the anti-flagella or the new anti-IR 6 tests. BBK32 variant antigens combined or in parallel may improve the accuracy of serology of EM. Ongoing studies are aimed at optimization of the antigenantibody conditions in BBK32 ELISA in order to improve differentiation between samples from EM patients and from controls.

\section{ACKNOWLEDGEMENTS}

This work was supported by the National Technology Agency (Tekes), Finland, the Foundation for Pediatric Research, Finland, and the Helsinki Central Hospital Research Funds, Finland.

We are grateful to Professor Allen Steere, of Harvard Medical School, for providing us with sera from US patients with EM.

\section{REFERENCES}

Aguero-Rosenfeld, M. E., Nowakowski, J., Bittker, S., Cooper, D., Nadelman, R. B. \& Wormser, G. P. (1996). Evolution of the serologic response to Borrelia burgdorferi in treated patients with cultureconfirmed erythema migrans. J Clin Microbiol 34, 1-9.

Fikrig, E., Feng, W., Barthold, S. W., Telford, S. R., III \& Flavell, R. A. (2000). Arthropod- and host-specific Borrelia burgdorferi bbk32 expression and the inhibition of spirochete transmission. J Immunol 164, $5344-5351$.

Gomes-Solecki, M. J., Wormser, G. P., Persing, D. H., Berger, B. W., Glass, J. D., Yang, X. \& Dattwyler, R. J. (2001). A first-tier rapid assay for the serodiagnosis of Borrelia burgdorferi infection. Arch Intern Med 161, $2015-2020$

Heikkilä, T., Seppälä, I., Saxen, H., Panelius, J., Peltomaa, M., Julin, T., Carlsson, S.-A. \& Lahdenne, P. (2002). Recombinant BBK32 protein in serodiagnosis of early and late Lyme borreliosis. J Clin Microbiol 40, $1174-1180$.

Huppertz, H.-I., Böhme, M., Standaert, S. M., Karch, H. \& Plotkin, S. A. (1999). Incidence of Lyme borreliosis in the Wurzburg region of Germany. Eur J Clin Microbiol Infect Dis 18, 697-703.

Junttila, J., Peltomaa, M., Soini, H., Marjamäki, M. \& Viljanen, M. K. (1999). Prevalence of Borrelia burgdorferi in Ixodes ricinus ticks in urban recreational areas of Helsinki. J Clin Microbiol 37, 1361-1365.

Liang, F. T., Steere, A. C., Marques, A. R., Johnson, B. J. B., Miller, J. N. \& Philipp, M. T. (1999). Sensitive and specific serodiagnosis of Lyme disease by enzyme-linked immunosorbent assay with a peptide based on an immunodominant conserved region of Borrelia burgdorferi VlsE. J Clin Microbiol 37, 3990-3996.

Mitchell, P. D., Reed, K. D., Aspeslet, T. L., Vandermause, M. F. \& Meslki, J. W. (1994). Comparison of four immunoserologic assays for detection of antibodies to Borrelia burgdorferi in patients with culturepositive erythema migrans. J Clin Microbiol 32, 1958-1962.

Nowakowski, J., Schwartz, I., Liveris, D. \& 7 other authors (2001). 
Laboratory diagnostic techniques for patients with early erythema migrans: a comparison of different techniques. Clin Infect Dis 33, 2023-2027.

Oksi, J., Marttila, H., Soini, H., Aho, H., Uksila, J. \& Viljanen, M. K. (2001). Early dissemination of Borrelia burgdorferi without generalized symptoms in patients with erythema migrans. APMIS 109, 581-588.

Seppälä, I. J. T., Kroneld, R., Schauman, K., Forsen, K. O. \& Lassenius, R. (1994). Diagnosis of Lyme borreliosis: non-specific serological reactions with Borrelia burgdorferi sonicate antigen caused by IgG2 antibodies. J Med Microbiol 40, 293-302.

Smith, R. P., Schoen, R. T., Rahn, D. W., Sikand, V. K., Nowakowski, J., Parenti, D. L., Holman, M. S., Persing, D. H. \& Steere, A. C. (2002).
Clinical characteristics and treatment outcome of early Lyme disease in patients with microbiologically confirmed erythema migrans. Ann Intern Med 136, 421-428.

Steere, A. C. (2001). Lyme disease. N Engl J Med 345, 115-125.

Strle, F., Nelson, J. A., Ruzic-Sabljic, E. \& 7 other authors (1996). European Lyme borreliosis; 231 culture-confirmed cases involving patients with erythema migrans. Clin Infect Dis 23, 61-65.

Vaz, A., Glickstein, L., Field, J. A., McHugh, G., Sikand, V. K., Damle, N. \& Steere, A. C. (2001). Cellular and humoral immune responses to Borrelia burgdorferi antigens in patients with culture-positive early Lyme disease. Infect Immun 69, 7437-7444. 